05

\title{
Неупругое растяжение медного однопроволочного проводника при неограниченных местных деформациях и положительной температуре
}

\author{
(C) А.И. Недобитков, Б.М. Абдеев
}

Восточно-Казахстанский технический университет им. Д. Серикбаева, 070014 Усть-Каменогорск, Казахстан

e-mail: a.nedobitkov@mail.ru

Поступило в Редакцию 30 сентября 2020 г.

В окончательной редакции 3 декабря 2020 г.

Принято к публикации 23 декабря 2020 г.

\begin{abstract}
Представлены результаты экспериментальных и теоретических исследований деформации однопроволочного медного проводника под действием токовой перегрузки. Проводник исследовался с помощью растрового электронного микроскопа JSM-6390L. На основе классической нелинейной задачи строительной механики получена математическая модель напряженно-деформированного состояния растянутого медного стержня при температуре до $700^{\circ} \mathrm{C}$. Определены механические усилия в однопроволочном медном проводнике, вызывающие образование шейки при протекании сверхтока. Математическая модель доведена до простых аналитических зависимостей, что позволяет использовать их при проведении судебной пожарно-технической экспертизы.
\end{abstract}

Ключевые слова: сила, напряжение, несущая способность, деформация, пластичность, диаграмма, медный проводник, токовая перегрузка.

DOI: $10.21883 / J T F .2021 .06 .50864 .282-20$

\section{Введение}

В работах $[1,2]$ отмечается, что при установлении причины пожара исследованию электропроводки на предмет наличия следов протекания аварийных процессов традиционно уделяется особое внимание. Авторами [2] показано, что потенциальная пожарная опасность электропроводки обусловлена сочетанием в ней горючей среды (электроизоляция, оболочки кабелей и т.п.) и источников зажигания (искры, дуги, нагретые электрическим током детали и т.п.), появляющихся при работе электрооборудования в аварийных режимах.

Так же в работах $[1,2]$ указывается, что при определенных значениях кратности перегрузки может произойти фрагментация (разделение на части медного проводника), а также появление на его поверхности характерных следов - утолщений, утончений (шеек) и вздутий. При этом в работе [2] приводятся экспериментальные данные при токе перегрузки 120-600 А с напряжением 220 V, и констатируется, что при кратности токовой перегрузки от 4 до 16 разрыв проводника происходит в результате плавления проводника, а также под действием сил поверхностного натяжения на медном проводнике образуются утолщения и утончения - шейки. А при кратности токовой перегрузки от 12 и выше разрыв проводника происходит в результате пинч-эффекта [2]. Некоторые аспекты поведения медных проводников под действием токовой перегрузки приведены в работе [3], наглядно подтверждающей наличие деформации в виде шеек.
С другой стороны, в работе [4] приводятся данные, согласно которым на поверхности медных образцов диаметром $1 \mathrm{~mm}$. при токе 50-2500 А возникают напряжения $(0.5-7) \cdot 10^{4} \mathrm{~Pa}$. Указанная величина может влиять на пластическую деформацию металлических кристаллов, но не определяет всей величины эффекта действия тока [4]. Авторами [4] показано, что расчетная максимальная осевая сила меньше в 25-30 раз наблюдаемых экспериментально скачков деформирующего усилия. Таким образом, „эффект пасты“, связанный с пинчдействием импульсного тока в опытах не имел места [4].

В свою очередь, в работе [5] исследовалась зона разрушения образца ультрамелкодисперсной меди при одноосном растяжении. Авторы [5] пришли к выводу, что работа тока во внешнем электрическом поле определяет производство энтропии, которое стабилизирует развитие локализованных полос сдвига с образованием в зонах пластических ротаций концентраторов напряжений. Фрагментация материала в зонах пластических ротаций вызывает рост величины деформирующих напряжений. Трансляционные сдвиги создают новые пластические ротации, и электропластический эффект обусловливает модуляцию внешних деформирующих напряжений подобно эффекту Портевена-Ле Шателье [5]. Несомненно, работа [5] имеет глубокое теоретическое значение, но в практике расследования причины пожаров мало применима.

В свете изложенного целью настоящей работы является определение механических усилий в однопроволочном медном проводнике, вызывающих образование 

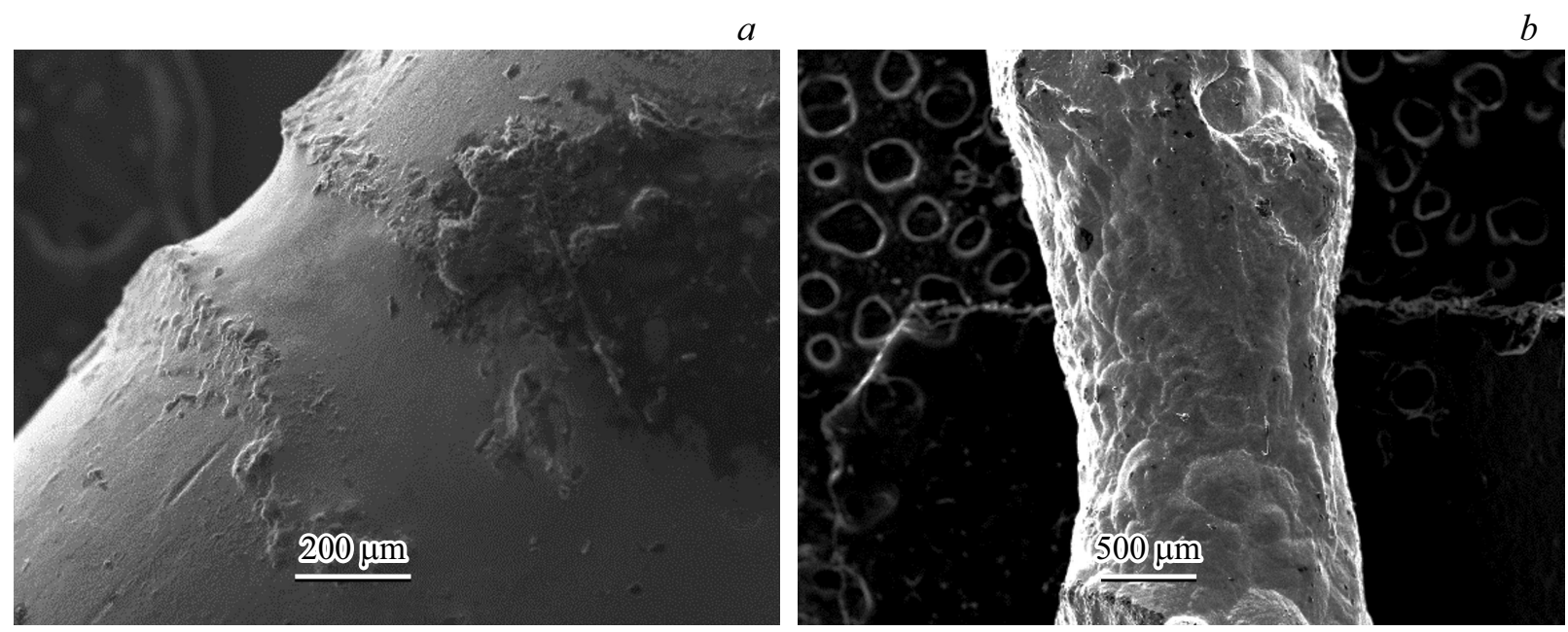

Рис. 1. Образование шейки медного проводника под действием токовой перегрузки: $a-$ начальный момент образования шейки, $b$ - момент перед фрагментацией, когда шейка истончена и близка к разрушению.

шейки при протекании сверхтока. Исходя из этого были поставлены следующие задачи исследования:

- вывести усовершенствованное решение классической нелинейной задачи строительной механики по математическому моделированию напряженно-деформированного состояния растянутого медного стержня в условиях положительного температурного градиента и теоретически неограниченных перемещений;

- показать, что не применяемая ранее степенная функциональная зависимость между условным напряжением $\sigma$ и деформацией $\varepsilon$, базирующаяся на четырех известных физико-механических константах $E, \sigma_{p p}, \sigma_{02}$, $\delta$, дает возможность с достаточной точностью как в количественном, так и качественном отношениях аппроксиммировать весь процесс неупругого статического растяжения, где для меди [6-8] $E$ - модуль упругости, $\sigma_{p p}-$ предел прочности, $\sigma_{02}-$ условный предел текучести, $\delta$ - остаточное относительное удлинение;

- довести алгоритм решения специальной задачи сопротивления материалов до простых конечных аналитических зависимостей, проиллюстрированных характерным примером расчета медного стержня круглого поперечного сечения при $t=20$ и $700^{\circ} \mathrm{C}$;

- продемонстрировать, что полученные результаты возможно непосредственно использовать при проведении судебных пожарно-технических экспертиз.

\section{1. Материалы и методика экспериментов}

Исследования выполнены в Центре превосходства „Veritas“ Восточно-Казахстанского технического университета им. Д. Серикбаева на растровом электронном микроскопе JSM-6390LV. Объектом исследования являлся однопроволочный медный проводник без изоляционного покрытия диаметром $1 \mathrm{~mm}$ и сечением $0.785 \mathrm{~mm}^{2}$.
Согласно справочным данным, максимально допустимая сила тока для медного проводника такого сечения составляет порядка 15-19 А. Эксперимент проводился по аналогии с работами $[1,2]$. Отличием от работ $[1,2]$ являлось то, что через проводник пропускали ток кратностью 4-6 не до его разрушения (фрагментации) [1,2], а до момента образования шейки и ее развития (рис. 1). На рис. 1 показаны различные стадии процесса образования шейки медного проводника под действием токовой перегрузки.

\section{2. Теоретические основы}

Для разработки математической модели напряженного состояния неупругого растяжения [6-9] медного стержня круглого поперечного сечения нагруженного силой $P$ в области наибольшего местного сужения (образования шейки) до момента предшествующего разрыву $[8,10]$ использовалась расчетная схема, приведенная на рис. 2. На расчетных схемах рис. 2 использованы следующие обозначения: $d x_{0}-$ бесконечно малый начальный слой материала стержня диаметром $d_{0} ; d x-$ продольный размер шейки, имеющей минимальное сечение $d$ при $x=0$, в процессе ее возникновения под действием нагрузки $P$.

Рассмотрим монотонное увеличение отрезка $d x_{0}$ до заметно большего значения $d x \gg d x_{0}$, когда $P \neq 0$. В геометрическом аспекте это свойство растянутого бруса характеризуется мерой, предложенной Коши $[8,11]$ :

$$
\varepsilon=\frac{d x-d x_{0}}{d x_{0}}=\frac{d x}{d x_{0}}-1=\frac{\Delta d x_{0}}{d x_{0}},
$$

где $\Delta d x_{0}=d x-d x_{0}-$ абсолютное удлинение участка $d x_{0}$. 


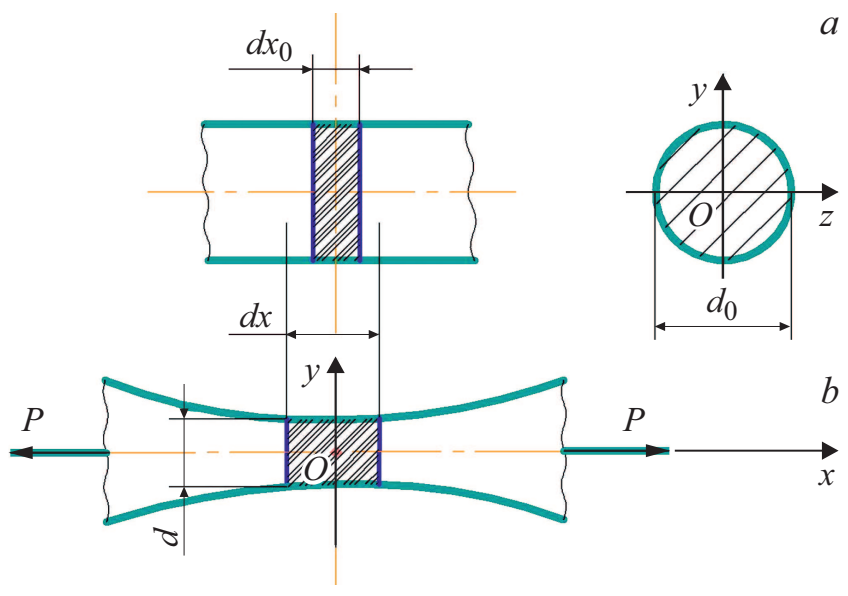

Рис. 2. Расчетная схема: $a-$ исходное состояние $(P=0)$, $b$ - после приложения силы $P$.

Безразмерный параметр $\varepsilon$ возможно применять тогда, когда $\varepsilon \leq 0.005$. В случае $\varepsilon \gg 0.005$, который охватывает весь процесс растяжения вплоть до образования шейки (рис. 2) и разрушения материала, необходимо переходить к так называемой истинной $\varepsilon_{i}$ или логарифмической деформации Генки $[8,11]$ :

$$
\varepsilon_{i}=\ln \left(\frac{d x}{d x_{0}}\right)=\ln \left(\frac{d x_{0}+\Delta d x_{0}}{d x_{0}}\right)=\ln (1+\varepsilon)<\varepsilon .
$$

При малых $\varepsilon \leq 0.005$ формулы (1) и (2) практически совпадают. Если же $\varepsilon>0.01$, то расхождение между $\varepsilon_{i}$ и $\varepsilon$ может быть заметным, а при $\varepsilon>0.1$ - существенным [8].

Погрешность $\Delta$ приближенного соотношения (1) определяется неравенством [8]

$$
\Delta=\left|\frac{\varepsilon-\varepsilon_{i}}{\varepsilon}\right|<\frac{1}{2}|\varepsilon|,
$$

которое справедливо как при растяжении $\left(\varepsilon, \varepsilon_{i}>0\right)$, так и в условиях сжатия $\left(\varepsilon, \varepsilon_{i}<0\right)$.

Актуальность поставленной задачи заключается, прежде всего, в возможности количественно оценивать несущую способность (прочность, жесткость, устойчивость $[8,11]$ ) растянутых стержневых элементов конструкций, обладающих физической и геометрической нелинейностью $[6,9,11,12]$. При этом следует подчеркнуть, что знание о величинах характеристик прочности, жесткости и пластичности конкретного материала в условиях нормальной (комнатной) температуры $t_{n}=20^{\circ} \mathrm{C}$ является уже далеко недостаточным для расчетов несущих конструкций, работающих с положительными и большими тепловыми перепадами (градиентами) $[7,11-16]$.

$$
T=t-t_{n}=t-20^{\circ} \mathrm{C} \geq 0 .
$$

Следует отметить, что с повышением температуры $t$ начальный модуль упругости $E=E(t)$, предел прочности $\sigma_{p p}=p p(t)$ и условный предел текучести $\sigma_{02}=\sigma_{02}(t)$ непрерывно и сильно понижаются у цветных металлов (медь, алюминий, свинец, цинк, никель) их сплавов [7,11,14-17].

В основу физико-математической модели одноосного неупругого растяжения (рис. 2) положим классические предпосылки прикладной механики изотропного однородного твердого тела $[7,8]$, и введем с учетом больших относительных линейных деформаций (2):

- в пределах размеров $d x, d x_{0}$ диаметры $d_{0}=$ const, $d=$ const, т.е. предполагаем, что в самом узком месте сужения сохраняется цилиндрическая форма [7-10,14];

- соблюдается гипотеза плоских сечений $[8,11,14]$;

- объем участков стержня длиной $d x, d x_{0}$ остается неизменным, что - характерно для пластичных материалов (в том числе меди) и равносильно коэффициенту Пуассона $[6,8,10,18] \mu=0.5$, что экспериментальнотеоретически подтверждено при испытаниях и расчетах лабораторных образцов, при условии $\varepsilon>0.02$ [8];

- вследствие малости коэффициента линейного теплового расширения меди $\alpha \ll 1[11,12,14]$ :

a) не меняются исходный $d_{0}$ и текущий (конечный) $d$ диаметры стержня при воздействии $t>20^{\circ} \mathrm{C}$;

б) соотношение между температурной деформацией $\varepsilon_{t}=\alpha T$ и нормальным напряжением $\sigma_{t}$ является линейным $[6,11]$

$$
\sigma_{t}=\sigma_{t}(t)=-E(t) \varepsilon_{t}(t)=-E(t) \alpha T
$$

и, принимая во внимание статическую определенность решаемой задачи при условии отсутствия кинематических связей, стесняющих свободное перемещение границ стержня, можно считать, что $\sigma_{t}=0$ и в этом случае напряженно-деформированное состояние будет характеризоваться только внутренними силовыми $\sigma, \sigma_{i}$ и геометрической $\varepsilon$ составляющими (рис. 3) [7,8,11,14].

На графиках рис. 3 использованы следующие обозначения: $\sigma, \varepsilon-$ условное нормальное напряжение $\sigma$ и соответствующая ему относительная растягивающая деформация $\varepsilon ; \beta$ - угол между касательной в точке „о“ и осью $\varepsilon ; \sigma_{i}-$ истинное напряжение; $\sigma_{p p}, \sigma_{i p p}-$ соответственно условный $\sigma_{p p}$ и фактический (истинный) $\sigma_{i p p}$ пределы прочности упруго-пластичного материала (меди); В, ВI - точки, соответствующие моменту действия наибольшей растягивающей силы $P_{\max }$, а также напряжениям $\sigma_{p p}, \sigma_{i p p}$ и началу возникновения шейки; $\varepsilon_{p p}$ - суммарная относительная деформация, соответствующая напряжениям $\sigma_{p p}, \sigma_{i p p} ; \delta-$ экспериментально определяемая величина остаточного относительного удлинения; C, CI, $\sigma_{c}, \sigma_{c I}$ - точки и напряжения $\left(\sigma_{c}, \sigma_{c I}\right)$, соответствующие деформации $\delta ; \varepsilon_{b}-$ пластическая (остаточная) часть полной деформации $\varepsilon_{p p}$.

Руководствуясь прогнозируемым очертанием условной диаграммы $\sigma-\varepsilon$ (рис. 3), в качестве аппроксимирующей функции $\sigma(\varepsilon)$ принимаем, учитывая $(2)$, оригинальную экспоненциально-логарифмическую степенную 


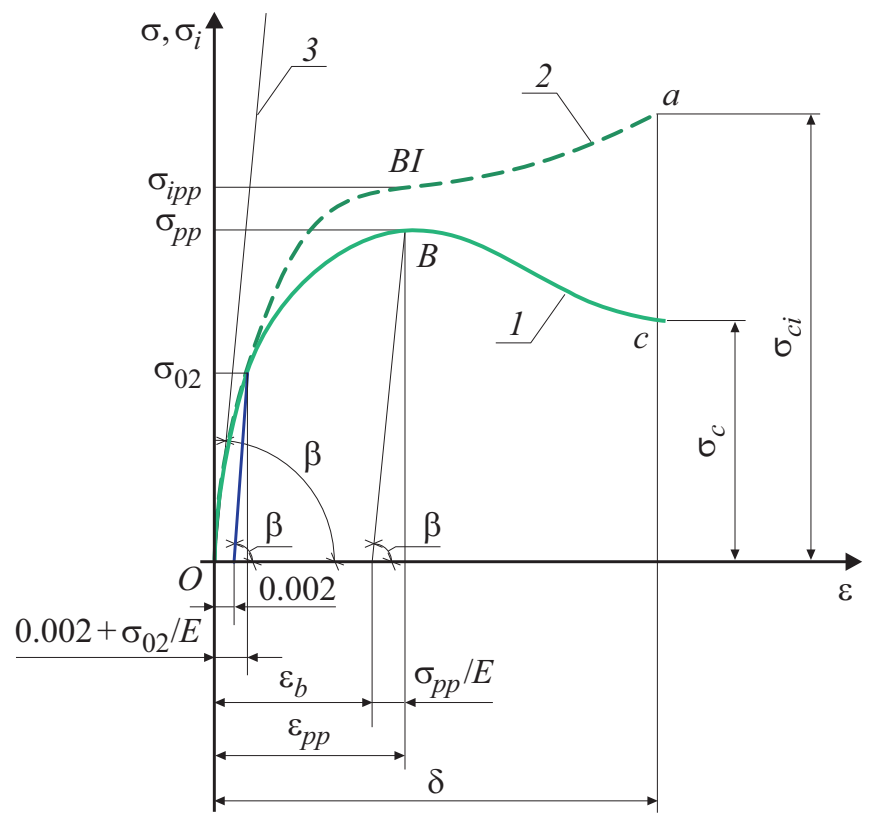

Рис. 3. Характер изменения основной моделирующей функции (6): 1 - условная диаграмма $\sigma(\varepsilon), 2$ - истинная зависимость $\sigma_{i}(\varepsilon), 3$ - касательная в точке „OI“ с тангенсом угла наклона $\operatorname{tg} \beta=E$.

зависимость $[18,19]$

$$
\begin{gathered}
\sigma=\sigma\left(\varepsilon_{i}\right)=A e^{-k \varepsilon_{\text {и }}^{n}} \varepsilon_{i}=\sigma(\varepsilon)=A e^{-k \ln ^{n}(1+\varepsilon)} \ln (1+\varepsilon), \\
0 \leq \varepsilon \leq \delta
\end{gathered}
$$

имеющую нисходящий участок, характерный для пластичных материалов [6-8], и экстремум, равный известному нормативному пределу прочности $\sigma_{p p}$, соответствующему искомой деформации

$$
\varepsilon_{p p}=\varepsilon_{t}+\frac{\sigma_{p p}}{E},
$$

где $A>0, k>0,0<n<1-$ параметры, подлежащие вычислению; $\delta=\delta\left(t^{\circ} \mathrm{C}\right)-$ средняя величина условного остаточного относительного удлинения [7,8,11,13] после разрушения стандартного лабораторного образца материала; $E=E(t)$ - начальный модуль упругости меди в зависимости от температуры $t[6,8,15] ; \varepsilon_{t}-$ остаточная деформация, адекватная временному сопротивлению $\sigma_{p p} ; e=2.71828$ - основание натурального логарифма [19].

Моменты действия максимального усилия $P_{\max }$ (рис. 2) соответствуют точкам В и ВI на обычной кривой деформирования 1 и истинной диаграмме 2 (рис. 3). При этом в граничной точке В на основании принятых допущений происходит потеря устойчивости по длине $d x$, равномерной пластической деформации, и начало возникновения шейки $[8,11]$.

Растягивающая сила [11] (рис. 2)

$$
P=\sigma_{i} F,
$$

где $F-$ фактическая площадь поперечного сечения:

$$
F=\frac{\pi d^{2}}{4}
$$

которая меньше начальной

$$
F_{0}=\frac{\pi d_{0}^{2}}{4}
$$

ввиду поперечных истинных деформаций $\varepsilon_{p i}$, связанных с $\varepsilon_{i}$ логарифмической зависимостью Пуассона $[8,10,11]$

$$
\varepsilon_{p i}=-\mu \varepsilon_{i}
$$

или, учитывая (2) и условие не сжимаемости материала (5) [10],

$$
\ln \frac{d}{d_{0}}=-0.5 \ln \left(\frac{d x}{d x_{0}}\right)=\ln \left(\frac{d x}{d x_{0}}\right)^{-1 / 2}
$$

откуда получаем

$$
\frac{d}{d_{0}}=\frac{1}{\left(\frac{d x}{d x_{0}}\right)^{1 / 2}}=\frac{1}{(1+\varepsilon)^{1 / 2}}
$$

При любой форме сечения

$$
\frac{F}{F_{0}}=\frac{4 \pi d^{2}}{4 \pi d_{0}^{2}}=\frac{1}{1+\varepsilon}, \quad F=\frac{F_{0}}{1+\varepsilon}
$$

на основании (10), (11) и (14).

Очевидно, что при критической нагрузке $P_{\max }$, согласно $(10)$, и на основании $[8,10,19]$ получаем равенства (рис. 2)

$$
d P=d \sigma_{i} F+\sigma_{i} d F=0, \quad\left[\frac{d P}{d \varepsilon}\right]_{\varepsilon=\varepsilon_{p p}}=0 .
$$

Для определения четырех констант $A, k, n, \varepsilon_{p p}$, входящих в формулы (6), (8), составляем 4 условия $[6,8]$.

1) Равенство предела прочности аппроксимирующей кривой (6) справочному экспериментальному значению $\sigma_{p p}$ при $\varepsilon=\varepsilon_{p p}$ :

$$
\sigma_{p p}=A e^{-k \ln ^{n}\left(1+\varepsilon_{p p}\right)} \ln \left(1+\varepsilon_{p p}\right) .
$$

2) Требование существования максимума

$$
\sigma_{\max }=\sigma_{p p}
$$

в точке В диаграммы „ $\sigma-\varepsilon^{“}$ с пока неизвестной абсциссой $\varepsilon_{p p}$ (рис. 3):

$$
\left[\frac{d \sigma}{d \varepsilon}\right]_{\varepsilon=\varepsilon_{p p}}=\left[E_{\mathrm{K}}(\varepsilon)\right]_{\varepsilon=\varepsilon_{p p}}=E_{\mathrm{K}}\left(\varepsilon_{p p}\right)=0,
$$

где $E_{\mathrm{\kappa}}(\varepsilon)-$ касательный модуль $[6,8,19]$. 
3) Равенство условного (опытного) предела текучести $\sigma_{02}[7,8,11]$ и моделирующего функционального соотношения (6), т.е. (рис. 3)

$$
\sigma_{02}=A e^{-k \ln ^{n}\left(1+\varepsilon_{\text {ost }}+\frac{\sigma_{02}}{E}\right)}\left(1+\varepsilon_{\text {ost }}+\frac{\sigma_{02}}{E}\right),
$$

когда остаточная пластическая деформация $\varepsilon_{o s t}=0.002$.

4) Равенство касательного модуля $E_{\mathrm{K}}$ начальному модулю упругости $E=E\left(t^{\circ} \mathrm{C}\right)$ в случае $\varepsilon=0$

$$
E_{\mathrm{K}}^{(0)}=\left[\frac{d \sigma}{d \varepsilon}\right]_{\varepsilon=0}=E=\text { const },
$$

выражающее автоматическую трансформацию функции (6) в классический закон Гука $[7,8,11,14] \sigma=E \varepsilon$.

С целью реализации условий (19), (21) записываем общее выражение первой производной [19]:

$$
\begin{aligned}
& \frac{d \sigma}{d \varepsilon}=\left[-k e^{-k \ln ^{n}(1+\varepsilon)} \frac{1}{1+\varepsilon} n \ln ^{n-1}(1+\varepsilon) \ln (1+\varepsilon)\right. \\
& \left.+e^{-k \ln ^{n}(1+\varepsilon)} \frac{1}{1+\varepsilon}\right]=\frac{A}{1+\varepsilon} e^{-k \ln ^{n}(1+\varepsilon)}\left[1-k n \ln ^{n}(1+\varepsilon)\right],
\end{aligned}
$$

где при $\varepsilon=0$ на основании (21)

$$
\left[\frac{d \sigma}{d \varepsilon}\right]_{\varepsilon=0}=A=E,
$$

а из равенства (19) находим соотношение

$$
k=\frac{1}{n \ln ^{n}\left(1+\varepsilon_{p p}\right)} .
$$

Подставляя (23), (24) в формулу (17) получаем

$$
\sigma_{p p}=E e^{-1 / n} \ln \left(1+\varepsilon_{p p}\right),
$$

откуда следует

$$
\varepsilon_{p p}=e^{\frac{\sigma_{p p}}{E} e^{1 / n}}-1 .
$$

Далее заменяем параметры $A, k, \varepsilon_{p p}, \varepsilon_{o s t}$ в аналитическом выражении (20), воспользовавшись условием $\varepsilon_{o s t}=0.002$ и выражениями (23)-(25). В итоге будем иметь трансцедентное уравнение [19] относительно $n$ вида

$$
\frac{1}{n}\left(\frac{0.002+\frac{\sigma_{02}}{E}}{\frac{\sigma_{p p}}{E}}\right)^{n}=-e \ln \frac{\sigma_{02}}{E\left(0.002+\frac{\sigma_{02}}{E}\right)}
$$

с известной правой частью.

В связи с указанной особенностью расчета показателя степени $n$ записываем условную диаграмму растяжения (6) в численном виде с интервалом (7) изменения деформации $\varepsilon$ для характерных тепловых режимов $t=20^{\circ} \mathrm{C}, t=700^{\circ} \mathrm{C}$ на примере стандартной электротехнической меди марки М1 по ГОСТ 859-2014 и ТУ16.К71-087-90, у которой $[15,16]$ :
— при $t=20^{\circ} \mathrm{C}$,

$$
\begin{gathered}
E=12714 \mathrm{~kg} / \mathrm{mm}^{2}, \sigma_{p p}=22.44 \mathrm{~kg} / \mathrm{mm}^{2}, \\
\sigma_{02}=6.12 \mathrm{~kg} / \mathrm{mm}^{2}, \quad \delta=0.45 ;
\end{gathered}
$$

— при $t=700^{\circ} \mathrm{C}$,

$$
\begin{gathered}
E=9883 \mathrm{~kg} / \mathrm{mm}^{2}, \quad \sigma_{p p}=3.06 \mathrm{~kg} / \mathrm{mm}^{2}, \\
\sigma_{02}=1.02 \mathrm{~kg} / \mathrm{mm}^{2}, \quad \delta=0.71 .
\end{gathered}
$$

Для удобства последующих вычислений преобразуем исходную зависимость (6) с учетом (23), (24)

$$
\sigma=\sigma(\varepsilon)=E e^{-\frac{\left[\frac{\ln (1+\varepsilon)}{\ln \left(1+\varepsilon_{p} p\right.} n^{n}\right.}{n}} \ln (1+\varepsilon)
$$

и конкретизируем равенство (27), пользуясь справочными данными [15] и работой [11]:

一 при $t=20^{\circ} \mathrm{C} \frac{1.40392^{n}}{6}=1.63931 e=4.4561$,

— при $t=700^{\circ} \mathrm{C} \frac{6.78615^{n}}{n}=3.0135 e=8.1915$,

откуда находим методом подбора с точностью до пятизначащих цифр после запятой:

— при $t=20^{\circ} \mathrm{C}$,

$$
n=n\left(20^{\circ} \mathrm{C}\right)=0.24376,
$$

— при $t=700^{\circ} \mathrm{C}$,

$$
n=n\left(700^{\circ} \mathrm{C}\right)=0.16860 .
$$

Основываясь на результатах расчета (29), (30), справочных данных [15], работе [11] и формулах (26), (28), вычисляем для характерных тепловых режимов $t=20,700^{\circ} \mathrm{C}$ условной диаграммы растяжения медного стержня (рис. 3 ):

- при $t=20^{\circ} \mathrm{C}$ :

$$
\begin{gathered}
\sigma=\sigma(\varepsilon)=12714 e^{-7.0775[\ln (1+\varepsilon)]^{0.24376}} \ln (1+\varepsilon), \\
0 \leq \varepsilon \leq \delta=0.45 ; \\
\varepsilon_{p p}=e^{0.001765 e^{4.1024}}-1=0.11266, \\
\sigma_{\mathrm{B}}=\sigma\left(\varepsilon_{p p}\right)=\sigma_{p p}=22.44 \mathrm{~kg} / \mathrm{mm}^{2}, \\
\sigma_{c}=\sigma(\delta)=\sigma(0.45)=18.18 \mathrm{~kg} / \mathrm{mm}^{2} .
\end{gathered}
$$

— при $t=700^{\circ} \mathrm{C}$ :

$$
\begin{gathered}
\sigma=\sigma(\varepsilon)=9883 e^{-8.521126[\ln (1+\varepsilon)]^{0.1686}} \ln (1+\varepsilon), \\
0 \leq \varepsilon \leq \delta=0.71 ; \\
\varepsilon_{p p}=e^{0.0003096 e^{5.931198}}-1=0.12367, \\
\sigma_{\mathrm{B}}=\sigma\left(\varepsilon_{p p}\right)=\sigma_{p p}=3.06 \mathrm{~kg} / \mathrm{mm}^{2}, \\
\sigma_{c}=\sigma(\delta)=\sigma(0.71)=2.47 \mathrm{~kg} / \mathrm{mm}^{2} .
\end{gathered}
$$


Расчетные значения напряжений $\sigma=\sigma(\varepsilon)$ и $\sigma_{i}=\sigma_{i}(\varepsilon)$ к построению диаграмм (рис. 4)

\begin{tabular}{c|c|r|r|r|r|r|r|r|c|c}
\hline$\varepsilon$ & 0 & \multicolumn{1}{|c|}{0.05} & 0.1 & \multicolumn{1}{c|}{0.2} & 0.3 & 0.4 & 0.5 & 0.6 & 0.7 & 0.8 \\
\hline$\sigma\left(20^{\circ} \mathrm{C}\right) \mathrm{kg} / \mathrm{mm}^{2}$ & 0 & 20.92 & 22.40 & 21.64 & 20.18 & 18.80 & 17.60 & - & - & - \\
$\sigma\left(700^{\circ} \mathrm{C}\right) \mathrm{kg} / \mathrm{mm}^{2}$ & 0 & 2.88 & 3.05 & 3.01 & 2.89 & 2.77 & 2.66 & 2.56 & 2.48 & 2.40 \\
$\sigma_{i}\left(20^{\circ} \mathrm{C}\right) \mathrm{kg} / \mathrm{mm}^{2}$ & 0 & 21.97 & 24.64 & 25.97 & 26.23 & 26.32 & 26.40 & - & - & - \\
$\sigma_{i}\left(700^{\circ} \mathrm{C}\right) \mathrm{kg} / \mathrm{mm}^{2}$ & 0 & 3.02 & 3.36 & 3.61 & 3.76 & 3.88 & 3.99 & 4.10 & 4.22 & 4.32
\end{tabular}

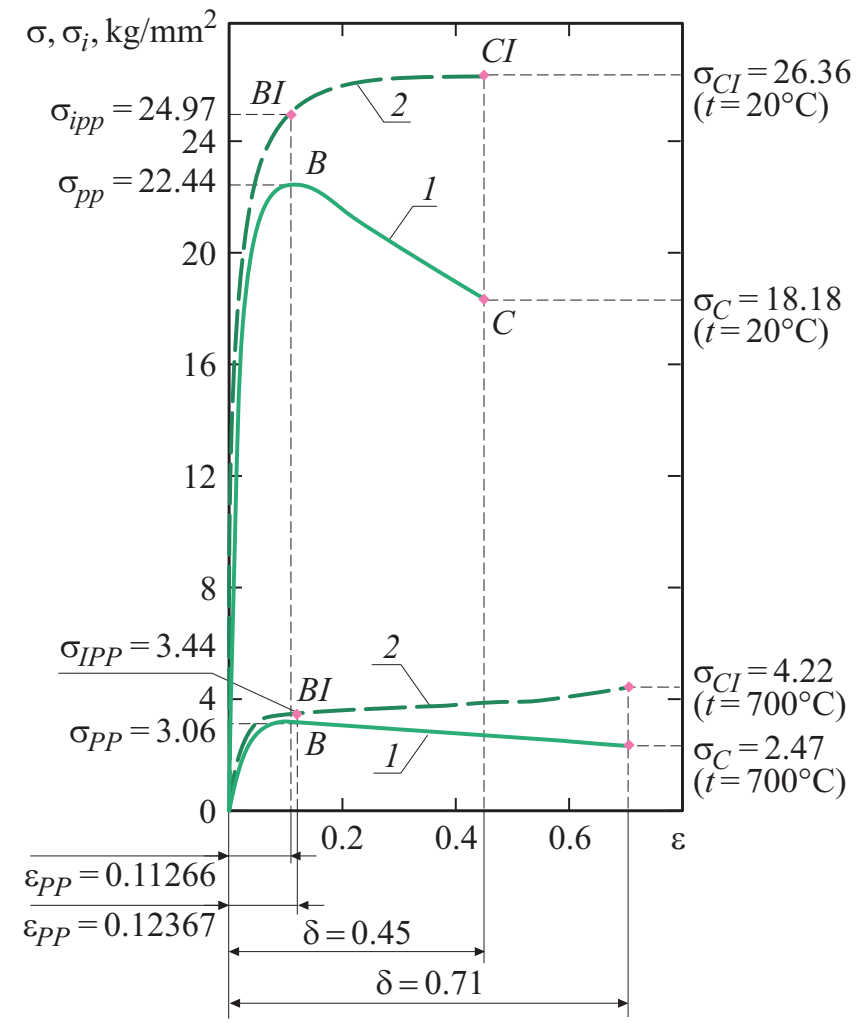

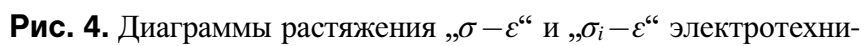
ческой меди: 1 - условные зависимости $\sigma(\varepsilon), 2-$ истинные функции $\sigma_{i}(\varepsilon)$.

На рис. 4 показаны функциональные зависимости (31), (34) в виде соответствующих графиков, построенных по данным таблицы с использованием основных численных характеристик (32), (33), (35), (36).

Следует отметить, что найденные граничные деформации $\varepsilon_{p p}\left(20^{\circ} \mathrm{C}\right)=0.11266$ и $\varepsilon_{p p}\left(700^{\circ} \mathrm{C}\right)=0.12367$ (см. (32) и (35) с учетом (8) вписываются в экспериментально установленный диапазон (рис. 3))

$$
\varepsilon_{b}=\varepsilon_{p p}-\frac{\sigma_{p p}}{E} \approx(0.1-0.4) \delta
$$

для пластичных материалов [8] и этот факт является одним из убедительных аргументов, подтверждающих физико-математическую корректность и точность предложенной диаграммы , $\sigma-\varepsilon^{“ 6}$ аппроксимируемой выражениями (26)-(28), где $\varepsilon_{b}$ - остаточная часть общей деформации $\varepsilon_{p p}$. С этой же точки зрения и характер поведения функции (28) в полной мере адекватен требуемому обобщенному виду, изображенному на рис. 3 и проиллюстрированному графиками рис. 4.

Кроме того, при $A=E>0, k>0,0<n<1$ кривые (6) и (28) асимптотически приближаются к оси $\varepsilon$ (рис. 3), т. е. согласно [19]:

$$
\lim _{\varepsilon \rightarrow \infty} \sigma(\varepsilon)=0
$$

что доказано результатами (23), (24), (26)-(36).

Всестороннее и детальное изучение процесса растяжения в условиях больших пластических деформаций $\varepsilon$ возможно только при совместном рассмотрении условной $\sigma(\varepsilon)$ и истинной $\sigma_{i}(\varepsilon)$ диаграмм $[7,8,11,13]$ (рис. 3). Для определения функции $\sigma_{i}(\varepsilon)$ предварительно записываем аналогичное выражению (10) соотношение $[8-11,14]$

$$
P=\sigma F_{0}
$$

а из очевидного равенства правых частей выражений (9) и (37), учитывая (15), находим

$$
\sigma_{i}=\sigma_{i}(\varepsilon)=\sigma \frac{F_{0}}{F}=\sigma(1+\varepsilon) .
$$

Графическая иллюстрация зависимости $\sigma_{i}(\varepsilon)$ показана штриховыми кривыми на рис. 4 для двух температур $t=20,700^{\circ} \mathrm{C}$ в соответствии с формулами (31), (34), (35) и расчетными данными таблицы. При этом фактические пределы прочности $\sigma_{i p p}\left(20^{\circ} \mathrm{C}\right), \sigma_{i p p}\left(700^{\circ} \mathrm{C}\right)$ и граничные напряжения $\sigma_{c i}\left(20^{\circ} \mathrm{C}\right), \sigma_{c i}\left(700^{\circ} \mathrm{C}\right)$, согласно (32), (33), (35), (36), (38), становятся, естественно, больше условных [8] одноименных силовых характеристик $\sigma_{p p}\left(20^{\circ} \mathrm{C}\right), \sigma_{p p}\left(700^{\circ} \mathrm{C}\right), \sigma_{c}\left(20^{\circ} \mathrm{C}\right), \sigma_{c}\left(700^{\circ} \mathrm{C}\right)$ (рис. 3, 4):

$$
\sigma_{i p p}=\sigma_{i}\left(\varepsilon_{p p}\right)=\sigma_{p p}\left(1+\varepsilon_{p p}\right)
$$

$$
\left.\begin{array}{c}
\sigma_{i p p}\left(20^{\circ} \mathrm{C}\right)=22.44(1+0.11266)=24.97 \mathrm{~kg} / \mathrm{mm}^{2}, \\
\sigma_{i p p}\left(700^{\circ} \mathrm{C}\right)=3.06(1+0.12367)=3.44 \mathrm{~kg} / \mathrm{mm}^{2}, \\
\sigma_{c i}=\sigma(\delta)(1+\delta), \\
\sigma_{c i}\left(20^{\circ} \mathrm{C}\right)=18.18(1+0.45)=26.36 \mathrm{~kg} / \mathrm{mm}^{2},
\end{array}\right\}
$$




\section{3. Расчеты}

В многопроволочных медных проводниках применяются проволочки диаметром $0.2 \mathrm{~mm}$, поэтому в расчетах используется эта величина. При этом диаметр $1 \mathrm{~mm}$ взят для сравнения.

В целях реализации возможности выполнения процедуры варьирования и регулирования несущей способности растянутого медного стержня $[8,10]$ необходимые для решения поставленной задачи результаты определяются по формуле

$$
P=P(\varepsilon)=\sigma F_{0}=\frac{\pi d_{0}}{4} \sigma(\varepsilon) .
$$

Наибольшее критическое усилие $P_{\max }$, соответствующее точке В на диаграмме рис. 4, выдерживаемое растянутым медным стержнем, согласно вышеприведенному алгоритму расчета, определяется по общей формуле

$$
P_{\max }=P\left(\varepsilon_{p p}\right)=\frac{\pi d_{0}}{4} \sigma_{p p}
$$

откуда находим при $d=1 \mathrm{~mm} P_{\max }\left(20^{\circ} \mathrm{C}\right)=17.62 \mathrm{~kg}$, $P_{\max }\left(700^{\circ} \mathrm{C}\right)=2.4 \mathrm{~kg}$, а при $d=0.2 \mathrm{~mm} P_{\max }\left(20^{\circ} \mathrm{C}\right)=$ $=0.705 \mathrm{~kg}, P_{\max }\left(700^{\circ} \mathrm{C}\right)=0.0961 \mathrm{~kg}$.

Когда $\varphi=\delta$, то в соответствии с (35), (37) при $d=1 \mathrm{~mm} P_{c}\left(20^{\circ} \mathrm{C}\right)=14.22 \mathrm{~kg}, P_{c}\left(700^{\circ} \mathrm{C}\right)=1.94 \mathrm{~kg}, \mathrm{a}$ при $d=0.2 \mathrm{~mm}$ соответственно, $P_{c}\left(20^{\circ} \mathrm{C}\right)=0.57 \mathrm{~kg}$, $P_{c}\left(700^{\circ} \mathrm{C}\right)=0.077 \mathrm{~kg}$.

Зная усилие, необходимое для образования шейки однопроволочного медного проводника, возможно по данным работы [4] определить диапазон силы тока, что в свою очередь, позволит установить причину как токовой перегрузки, так и пожара в целом. Проблема определения причины токовой перегрузки и ее величины остается актуальной и требует своего решения [20-22]. Приведенные расчеты хорошо согласуются с данными работы [3] и на практике подтверждаются отзывной компанией автомобилей Lada XRay из-за проблем с проводкой. Согласно сообщению пресс-служба Росстандарта, под отзыв попало 1278 автомобилей, реализованных с сентября 2019 по февраль 2020 г. На указанных автомобилях будет проверена правильность укладки проводки панели приборов.

\section{Заключение}

Предложенное решение классической нелинейной задачи строительной механики по математическому моделированию напряженно-деформированного состояния растянутого медного стержня в условиях положительного температурного градиента и теоретически неограниченных перемещений является оригинальным и уточненным по существу.

Подобранная и не применяемая ранее степенная функциональная зависимость между условным напряжением $\sigma$ и деформацией $\varepsilon$, базирующаяся на четырех известных физико-математических константах $E, \sigma_{p p}, \sigma_{02}, \delta$, дает возможность с достаточной точностью как в количественном, так и качественном отношениях аппроксиммировать весь процесс неупругого статического растяжения.

Найденная кривая $\sigma=\sigma(\varepsilon)$ легко преобразуется в истинную диаграмму $\sigma_{i}(\varepsilon)$ и функциональное соотношение $P(\varepsilon)$ между растягивающим усилием и условной деформацией $\varepsilon$, что представляется чрезвычайно важным при теоретической аппроксимации больших пластических деформаций и для расчета несущей способности стержня по нагрузке $P$ и ее экстремуму $P_{\max }$.

Алгоритм решения специальной задачи сопротивления материалов доведен до простых конечных аналитических зависимостей, проиллюстрированных характерным примером расчета медного стержня круглого поперечного сечения при $t=20,700^{\circ} \mathrm{C}$.

Полученные результаты возможно непосредственно использовать при проведении пожарно-технической экспертизы.

\section{Благодарности}

Микроскопические исследования были выполнены на оборудовании Центра превосходства „Veritas“ ВосточноКазахстанского технического университета им. Д. Серикбаева.

\section{Конфликт интересов}

Авторы заявляют, что у них нет конфликта интересов.

\section{Список литературы}

[1] А.Ю. Мокряк, И.Д. Чешко, В.В. Пеньков. Проблемы управления рисками в техносфере, 4 (32), 41 (2014).

[2] И.Д. Чешко, А.Ю. Мокряк, С.В. Скодтаев. Вестник СПбУ ГПС МЧС России, 1, 41 (2015).

[3] A.I. Nedobitkov. Fire and Explosion Safety, 28 (4), 42 (2019). (in Russian). DOI: 10.18322/PVB.2019.28.04.42-50

[4] В.И. Спицын, О.А. Троицкий. Электропластическая деформация металлов (Наука, М., 1985), 160 с.

[5] В.Е. Егорушкин, В.Е. Панин, А.В. Панин. Физическая мезомеханика, 3 (21), 5 (2018).

[6] П.А. Лукаш. Основы нелинейной строительной механи$\kappa и$ (Стройиздат, М., 1978), 204 с.

[7] А.П. Филин. Прикладная механика твердого дебормируемого тела. Сопротивление материалов с элементами теории сплошных сред и строительной механики (Наука, М., 1975), т. 1, 832 с.

[8] И.А. Биргер, Р.Р. Мавлютов. Сопротивление материалов: учебное пособие (Наука, М., 1986), 560 с.

[9] С.П. Тимошенко, Дж. Гере. Механика материалов / пер. с англ. Л.Г. Корнейчука, под ред. Э.И. Григолюка (Мир, М., 1976), 672 c.

[10] Я.Г. Пановко, И.Н. Губанова. Устойчивость и колебания упругих систем: современные конщепции, парадоксы и ошибки (Наука, М., 1987), 4-е изд., 352 с.

[11] В.И. Феодосьев. Сопротивление материалов (Наука, М., 1974), $500 \mathrm{c}$ 
[12] С.В. Серенсен, В.П. Когаев, В.Н. Шнейдерович. Несущая способность и расчет деталей машин на прочность: Рук-во и справочное пособие, под ред. Серенсена С.В. (Машиностроение, М., 1975), 488 с.

[13] И.А. Биргер, Б.Ф. Шорр, Г.Б. Иосилевич. Расчет на прочность деталей машин: справочник (Машиностроение, M., 1979), 702 c.

[14] Н.М. Беляев. Сопротивление материалов (Наука, М., $1965), 856 \mathrm{c}$.

[15] А.К. Николаев, С.А. Костин. Медь и жсаропрочные медные сплавы: энщикл. терминолог. слов: фундаментальный справ. (ДПК Пресс, М., 2012), 715 с.

[16] В.И. Сакало, Ю.С. Гусева, Т.В. Иншакова. Вестн. Брянского гос. тех. ун-та., 3 (47), 94 (2015).

[17] В. Новацкий. Вопросы термоупругости / пер. с польского (Изд-во АН СССР, М., 1962), 364 с.

[18] Л.М. Качанов. Основы теории пластичности (Наука, M., 1969), $420 \mathrm{c}$.

[19] И.Н. Бронштейн, К.А. Семендяев. Справочник по математике для инженеров и учащихся втузов (Физматлит, M., 1962), 608 c.

[20] V. Babrauskas. J. Fire Sci., 36, 438 (2018).

[21] V. Babrauskas. Rev. Fire Safety J., 89, 7 (2017).

[22] V. Babrauskas. Electrical Fires, in SFPE Handbook of Fire Protection Engineering, 5th ed. (Springer, NY., 2016), p. 662. 